\title{
Endocrine disrupting chemicals-how to strengthen safety assessment
}

Principles of endocrinology need to be applied to strengthen the safety assessment of endocrine disrupting chemicals (EDCs), a statement from the US Endocrine Society concludes.

"The Endocrine Society had received many requests from the US Congress on just how to apply the 2009 Scientific Statement on EDCs to legislation or to the public health domain," explains Thomas Zoeller of the University of Massachusetts Amherst, USA, one of a team of experts who put together the document. The new Statement of Principles central tenet is that EDCs cannot be evaluated as if they are general toxins. After all, EDCs interfere with hormone actions that are highly complex and tissue-specific, and that change over the lifetime of an individual.

The definition of an EDC is an essential starting point in the chemical safety assessment process. Various agencies have produced definitions of EDCs. The experts of the Statement of Principles propose the use of a simplified version of the definition from the US Environmental Protection Agency: "An EDC is an exogenous chemical, or mixture of chemicals, that interferes with any aspect of hormone action." Importantly, this definition includes mode of action but omits mention of causing harm, which is present in some other definitions but can only be properly assessed at a later stage of the process of safety evaluation. The experts consider the capacity of EDCs to interfere with hormone action as an inherent predictor of adverse outcome.

A classical toxicological approach, which is founded in the analysis of linear dose responses, will probably underestimate the potency of EDCs and miss important effects, the experts explain. Firstly, for EDCs, high-dose effects do not predict low-dose effects, as dose-response curves for these compounds, as for hormones, are sigmoidal or even nonmonotonic. Secondly, the effect of exposure to an EDC depends on life stage. Thus, a presumptive 'safe' dose might still have permanent effects if exposure occurs during a life stage in which no endogenous hormone is normally present. To add further complexity, the effects of EDCs often do not appear until many years after exposure. Angel Nadal of Miguel Hernández University of Elche, Spain, who was not involved in this statement, champions the recommendations in the statement by calling for a clear change in the

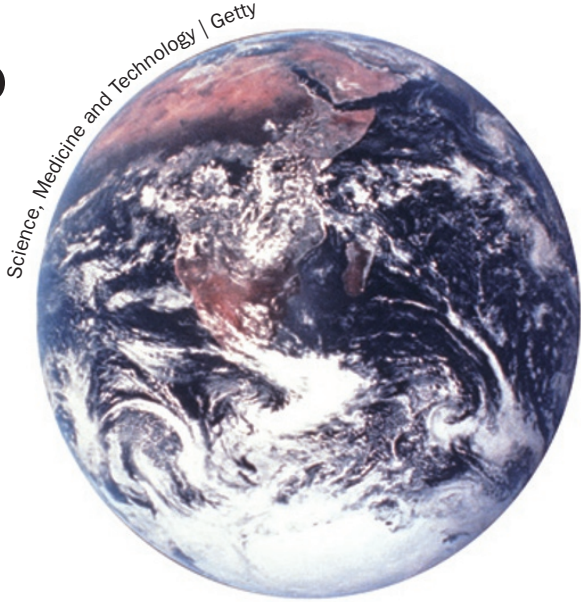

way hazard and risk assessments are performed. "Scientist working directly on EDCs with an endocrinology approach must be incorporated into evaluation panels. Evaluations must include full dose-response curves and establish the shape of those dose responses. Evaluation of several sensitive end points, sensitive time of exposure and visibility of effects is also required. These changes will make hazard and risk evaluation of EDCs more complicated but will certainly improve public health."

Carol Wilson

Original article Zoeller, R. T. et al. Endocrine-disrupting chemicals and public health protection: a statement of principles from the Endocrine Society. Endocrinology doi:10.1210/en.2012-1422 